\title{
Impact of Chronicity on Outcomes Following Post-Hospital Residential Brain Injury Rehabilitation: Application of Multivariate Statistics and Rasch Analysis
}

\author{
Frank D. Lewis ${ }^{1}$, Gordon J. Horn ${ }^{2}$, Robert Russell ${ }^{3}$ \\ ${ }^{1}$ NeuroRestorative Research Institute, Medical College of Georgia, Augusta University, Augusta, USA \\ ${ }^{2}$ NeuroRestorative Research Institute, College of Medicine, Florida State University, Orlando, USA \\ ${ }^{3}$ NeuroRestorative Research Institute, Benedictine University, Carbondale, USA \\ Email: frank.lewis@neurorestorative.com
}

How to cite this paper: Lewis, F.D., Horn, G.J. and Russell, R. (2017) Impact of Chronicity on Outcomes Following Post-Hospital Residential Brain Injury Rehabilitation: Application of Multivariate Statistics and Rasch Analysis. Open Journal of Statistics, 7, 254263.

https://doi.org/10.4236/ojs.2017.72020

Received: March 28, 2017

Accepted: April 23, 2017

Published: April 26, 2017

Copyright (๑) 2017 by authors and Scientific Research Publishing Inc. This work is licensed under the Creative Commons Attribution International License (CC BY 4.0).

http://creativecommons.org/licenses/by/4.0/

\begin{abstract}
This study evaluated the impact of chronicity (onset of injury to admission interval) on three domains of functional outcomes for a large group of traumatic brain injured (TBI) survivors. Subjects included 528 TBI adults who were treated in post-hospital residential rehabilitation centers. Subjects were assigned to one of three chronicity groups: 1) Early Interval (EI), $2.00-8.00$ months $\mathrm{n}=245,2)$ Mid Interval (MI), $8.01-24.00$ months $\mathrm{n}=129$, and (3) Late Interval (LI), 24.01 months and greater $n=154$. Functional status was assessed with the MPAI-4. RM MANCOVA was applied to evaluate differences among groups from admission to discharge. Rasch analysis demonstrated satisfactory construct validity and internal consistency (Person reliability = $0.90-0.94$, Item reliability $=0.99$ ) for the admission and discharge MPAI-4s. Controlling for LOS and age, the RM MANCOVA revealed that each chronicity group showed significant improvement in MPAI-4 abilities, adjustment, and participation indices from admission to discharge $(\mathrm{p}<0.001)$. Improvement observed from admission to discharge was the greatest among the EI group ( $\mathrm{p}<0.001)$. This study demonstrated the utility of multivariate statistical approaches for understanding the complexities of TBI treatment outcomes. As measured across three domains of functioning, rehabilitation was effective in reducing disability for participants in each chronicity group. Of the three groups, EI participants presented as the most disabled at admission but also made the greatest gains when assessed at discharge.
\end{abstract}

\section{Keywords}

TBI, Multivariate Analysis, Chronicity, Outcome, Post-Hospital 
Rehabilitation, MPAI-4, Raschanalysis, Functional Assessment

\section{Introduction}

An important question for treatment of traumatic brain injury (TBI) in posthospital rehabilitation settings is whether rehabilitation benefits chronically injured persons, those with a late onset to the admission interval. It is well documented that the functional change is the most rapid during the first six months following injury, due largely to the interaction of natural recovery and structured rehabilitation [1] [2]. However, recent studies of post-hospital TBI outcomes indicate that late interval rehabilitation (initiated one or more years post injury) may achieve the significant reduction in disability, although gains are achieved more slowly than for those who receive the early rehabilitation [3] [4].

In order to meaningfully evaluate the effectiveness of late interval rehabilitation, several challenges to TBI outcome measurement must be addressed. First, the heterogeneity of injury and outcome necessitate comprehensive measures across multiple domains to capture the extent of disability and detect the change in performance. A TBI may disrupt a few, or many, functions to varying degrees of severity. No single measure is sufficient to assess the potential breadth and complexity of impairment to physical, cognitive, emotional and social functioning [3].

Just as important, the measures selected must be psychometrically sound (established reliability and validity) and analyzed with appropriate statistics that will enable the most accurate interpretation of the data [5]. The majority of published TBI outcome studies have employed ordinal measures to evaluate program effectiveness [6]. Ordinal measures typically involve Likert scales that rank persons as possessing "a lot or a little" of a particular attribute. However, the intervals between levels on these scales are not equal, and therefore, not appropriate for use with parametric statistics involving summing and averaging numbers to calculate change scores [5] [6]. Nonetheless, TBI functional outcome literature is replete with studies applying parametric statistics to ordinal data [7]. This practice can lead to inaccurate inferences, either over- or under-estimating treatment effects [5] [6]. A more precise scientific assessment of program outcomes involves the use of interval measures appropriate for application with complex parametric statistics [8].

The purpose of this study was to evaluate the impact of chronicity (onset of injury to admission interval) on functional outcomes following post-hospital rehabilitation. To address the challenges of functional outcome measurement, three interval-level dependent measures derived from the Mayo Portland Adaptability Inventory-4 [9] were employed to assess the range of possible cognitive/ physical, emotional adjustment, and societal participation changes from program admission to discharge. The measures selected have strong reliability and validity data and are comprehensive enough to provide a clear indication of the 
problems experienced by TBI survivors [9]. To further establish item reliability and construct validity, Rasch analysis was performed on the measures at admission and discharge. Scores on the three measures were converted to T-scores, enabling the use of multivariate statistical approaches to identify and clarify the complex interactions among chronicity groups with time of measurement and skill domain.

\section{Methods}

\subsection{Subjects}

The study sample was selected from 2500 neurologically impaired individuals with consecutive discharges from 32 post-hospital residential rehabilitation programs in 15 states from 2011 to 2016. From the population of 2500, a sample of 528 individuals met study inclusion criteria: diagnosed with a traumatic brain injury, age 17 or older, minimum length of stay of 2 months, minimum chronicity (onset of injury to admission interval) of 2 months and admitted and discharged from active residential neurorehabilitation. The extent and nature of their disability prevented these participants from living independently. The severity of disability upon admission to program was assessed by MPAI-4 Total T-scores (a measure of disability compared to a referenced group of neurologically impaired persons) [7]. The mean length of stay for the entire sample was 8.9 months. The mean onset of injury to admission was 39.9 months. The average age for the total sample was 40.5 years. Detailed demographic characteristics of the sample including MPAI-4 Total T-scores at admission are presented in Table 1.

To examine the impact of chronicity on outcome, the study sample was divided into three groups based on the interval from onset of injury to rehabilitation admission: Early Interval (EI): $2.00-8.00$ months $(\mathrm{n}=245)$, Mid Interval (MI): $8.01-24.00$ months $(\mathrm{n}=129)$, and Late Interval (LI): 24.01 months and greater $(\mathrm{n}=154)$. Demographic characteristics of each group are presented in Table 2.

\subsection{Measure}

Participant functioning was assessed using the Mayo-Portland Adaptability Inventory-4 (MPAI-4) at the time of admission and time of discharge from the treatment facilities involved in the study. Specifically, the MPAI-4 consists of 29 items rated from 0 to 4 on a 5-point scale, where 0 represents no limitations and 4 represents a severe problem interfering with activity more than $75 \%$ of the time. Raw scores on the 29 items are converted to T-scores within three subscales: Abilities Index (physical, communication, and cognitive skills), Adjustment Index (emotional and behavioral skills), and Participation Index (contextual skill application). T-scores have a mean of 50 and a standard deviation of 10 . Higher T-scores indicate greater disability. Used primarily in post-hospital rehabilitation settings, the MPAI-4 has undergone rigorous psychometric testing and has proven reliability and validity as determined through Rasch analysis, Item 
Table 1. Total sample demographics and injury related variables, $\mathrm{n}=528$.

\begin{tabular}{|c|c|}
\hline Sample Demographics & Values \\
\hline \multicolumn{2}{|l|}{ Gender } \\
\hline Male & $82 \%$ \\
\hline Female & $18 \%$ \\
\hline \multicolumn{2}{|l|}{ Age (years) } \\
\hline Mean & 40.5 \\
\hline $\mathrm{SD}^{*}$ & 14.4 \\
\hline Range & $17-80$ \\
\hline \multicolumn{2}{|l|}{ Time Since Injury (months) } \\
\hline Mean & 39.9 \\
\hline SD & 77.6 \\
\hline Range & $2.0-564.6$ \\
\hline \multicolumn{2}{|l|}{ Length of Stay (months) } \\
\hline Mean & 8.9 \\
\hline $\mathrm{SD}$ & 10.5 \\
\hline Range & $2.0-67.4$ \\
\hline \multicolumn{2}{|l|}{ Race } \\
\hline African American & $11 \%$ \\
\hline Asian/Pacific & $1 \%$ \\
\hline Caucasian & $75 \%$ \\
\hline Hispanic & $9 \%$ \\
\hline Multi-racial & $4 \%$ \\
\hline \multicolumn{2}{|l|}{ Severity of TBI } \\
\hline Mild $(<40)$ & $1.0 \%$ \\
\hline Mild-moderate (40 - 49) & $15.0 \%$ \\
\hline Moderate (50 - 59) & $36.8 \%$ \\
\hline Severe $(60+)$ & $47.2 \%$ \\
\hline
\end{tabular}

${ }^{*} \mathrm{SD}=$ Standard Deviation calculated with the formula $\sigma=\sqrt{\frac{\sum(x-\mu)^{2}}{N}}$.

Cluster, Principle Component Analyses (PCA), and measures of concurrent and predictive validity [9] [10].

T-scores derived for the three MPAI- 4 indices at admission and discharge constituted the dependent variables for the study. For a detailed description of the 29 MPAI-4 items, Indices, and rating scale see Malec and Lezak 2008 [9].

\subsection{Rehabilitation Treatment}

Each participant was admitted to residential neurorehabilitation program where they received physical therapy, occupational therapy, speech therapy, recreation, counseling (based on need), case management, and medical management provided by nursing and physicians specializing in physical medicine and rehabilitation. Behavioral analysis was provided for cases requiring more extensive 
Table 2. Demographics by chronicity groups.

\begin{tabular}{|c|c|c|c|}
\hline & \multicolumn{3}{|c|}{ Chronicity Groups } \\
\hline & $\begin{array}{l}\text { Early Interval } \\
2 \text { - } 8 \text { Months } \\
\quad(\mathrm{n}=245)\end{array}$ & $\begin{array}{c}\text { Mid Interval } \\
8.01-24 \text { Months } \\
\quad(\mathrm{n}=129)\end{array}$ & $\begin{array}{l}\text { Late Interval } \\
24.01+\text { Months } \\
(\mathrm{n}=154)\end{array}$ \\
\hline \multicolumn{4}{|l|}{ Age } \\
\hline Mean & 42.4 & 39.6 & 38.12 \\
\hline $\mathrm{SD}$ & 15.3 & 13.7 & 13.0 \\
\hline Range & $18-80$ & $17-75$ & $18-68$ \\
\hline \multicolumn{4}{|l|}{ Gender } \\
\hline Male & $64 \%$ & $74 \%$ & $78 \%$ \\
\hline Female & $36 \%$ & $26 \%$ & $22 \%$ \\
\hline \multicolumn{4}{|c|}{ Onset to Admission (months) } \\
\hline Mean & 4.2 & 13.8 & 118.5 \\
\hline $\mathrm{SD}$ & 1.6 & 4.6 & 108.9 \\
\hline Range & $2-8$ & $8.01-24$ & $24.01-564.7$ \\
\hline \multicolumn{4}{|c|}{ Length of Stay (months) } \\
\hline Mean & 7.7 & 8.9 & 11.1 \\
\hline SD & 9.3 & 10.6 & 11.8 \\
\hline Range & $2-65.5$ & $2-67.4$ & $2-57.7$ \\
\hline \multicolumn{4}{|c|}{ Severity of TBI } \\
\hline Mild & $0.0 \%$ & $3.1 \%$ & $1.3 \%$ \\
\hline Mild-Mod & $13.1 \%$ & $16.3 \%$ & $16.9 \%$ \\
\hline Moderate & $31.8 \%$ & $31.8 \%$ & $48.7 \%$ \\
\hline Severe & $55.1 \%$ & $48.8 \%$ & $33.1 \%$ \\
\hline
\end{tabular}

modification to reduce inappropriate behaviors and increase positive replacement behaviors.

\subsection{Procedure}

Participants were evaluated upon admission by each program's multidisciplinary treatment team members. Once individual discipline assessments were completed, each participant was then evaluated within approximately two weeks of admission using the MPAI-4 by treatment team consensus. Discharge MPAI-4s were completed in a similar fashion within the final week of the participant's stay. The results of all evaluations were compiled into a national database and combined with participant demographic data.

\subsection{Statistical Analysis}

Rasch analysis was conducted for purposes of determining reliability and construct validity of the MPAI-4 as a measure of disability following brain injury. A repeated measures multivariate analysis of co-variance (RM MANCOVA) was provided to evaluate change scores on Abilities, Adjustment, and Participation Indices from admission to discharge. Differences in outcome as a function of chronicity group were determined using this statistical approach. Analyses were performed using SPSS version 22 for the RM MANCOVA and follow-up tests 
while Winsteps version 3.81 was used to conduct Rasch analyses.

\section{Results}

\subsection{Reliability and Validity of MPAI-4}

Rasch analysis determines the reliability of an assessment tool by comparing expected from the actual values of the items comprising a measure. The analysis reveals the extent of item and person fit for measurement of human performance. According to prior research this analysis "has been used to evaluate how items contributing to a measure represent the underlying construct and how well the items provide a range of indicators that reliably differentiate among people rated with the measure" [10]. Key statistics provided by Rasch analysis are Person and Item Reliability and Person and Item Separation. In general, reliability refers to the reproducibility of results obtained by a measure. Specifically, Person Reliability indicates how well items comprising a measure distinguish among individuals (e.g. those possessing a lot or a little of the construct measured) while Item Reliability refers to whether test items relate to each other in a consistent way in describing a disparate group of individuals. A coefficient of 0.80 or greater is considered acceptable for Person Reliability, while a coefficient of at least 0.90 is optimal for Item Reliability [8]. For the current sample, Person Reliability coefficients were 0.90 and 0.94 respectively for admission and discharge MPAI-4s. Item Reliability was 0.99 for both admission and discharge MPAI-4s.

Separation values indicate "the extent to which items distinguish among people (Person Separation) and are distinct from each other (Item Separation)" [10]. Specifically, Person Separation values indicate the number of performance levels detected by a measure. For example, a Person Separation index of 2.00 means that two levels of performance can be reliably identified. The present study found Person Separation indices of 2.95 and 3.85 respectively for admission and discharge MPAI-4s indicating 3.00+ levels of performance reliably identified. Item Separation refers to the extent to which items on a test are consistently ranked from least difficult to most difficult. Low Item Separation $(<3.00)$ implies that the item difficulty hierarchy is not reliable [8], whereas magnitudes exceeding 3.00 indicate greater consistency of item hierarchy. Item Separation indices for the study sample were 13.75 and 15.93 respectively for admission and discharge MPAI-4s, revealing a strong hierarchical item structure. With the reliability and construct validity of the MPAI-4 established, results were analyzed to determine the effect of age on outcome following post-hospital residential brain injury rehabilitation.

\subsection{Impact of Chronicity at Admission to Program}

A MANCOVA was performed on the three dependent variables (Abilities, Adjustment, and Participation Index scores) to examine differences in severity of disability among chronicity groups at the time of admission. Prior to interpreting those results, the Box's $M$ test was examined to assess the equality of cova- 
riance across the three chronicity groups. This result was non-significant ( $\mathrm{p}<$ 0.049 ; significance determined at alpha $=0.001$ ), meeting the assumption for multivariate homogeneity of variance (equality of variance between groups).

After controlling for age, the MANCOVA revealed a significant main effect for chronicity group, $\mathrm{F}(6,1042)=5.6, \mathrm{p}<0.0005$, Wilks Lambda $=0.939$, partial eta $^{2}=0.031$. Post hoc LSD comparisons found that on the Abilities and Participation indices, the LI group differed (lower scores, less disability) from both EI and MI chronicity groups ( $p<0.0005$ for Abilities, and $p<0.05$ for Participation).

\subsection{Change Admission to Discharge}

With length of stay and age entered as covariates, a RM MANCOVA revealed a significant main effect for pre-post testing, $\mathrm{F}(1,523)=91.78 \mathrm{p}<0.0005$, Wilks Lambda $=0.85$, partial eta ${ }^{2}=0.15$, power to detect $=1.00$. Follow-up paired sample t-tests indicated that within each chronicity group, scores on the MPAI-4 indices were significantly lower (less disability) from admission to discharge. Table 3 presents the paired sample T-values, significance levels, and Cohen's d effect sizes for each pre-post comparison on the Abilities, Adjustment, and Participation measures.

Results of the RM MANCOVA also showed a significant three way chronicity group by pre-post testing by measure interaction, $\mathrm{F}(2,523)=16.69, \mathrm{p}<0.0005$, Wilks Lambda $=0.94$, partial eta ${ }^{2}=0.06$. To interpret this interaction, first, post hoc LSD comparisons were performed revealing that mean differences admission to discharge on the three measures from pre to post testing were significantly greater for the EI group compared to the LI group, $\mathrm{p}<0.02$. The MI group did not differ significantly from the EI and LI groups. Next, post hoc one-way ANOVAs were conducted on the three MPAI-4 indices at discharge. This analysis found no significant differences among chronicity groups on any of the measures, indicating that group differences observed on these measures at admission were not present at discharge.

Taken together, these findings revealed that participants in each of the three

Table 3. Paired-sample T-values and effect sizes for change admission to discharge by chronicity groups on MPAI-4 indices.

\begin{tabular}{cccc}
\hline & \multicolumn{2}{c}{ Paired-sample T-Values (Cohen's d effect size) } \\
\hline Chronicity Group & $\begin{array}{c}\text { Abilities } \\
\text { Index }\end{array}$ & $\begin{array}{c}\text { Adjustment } \\
\text { Index }\end{array}$ & $\begin{array}{c}\text { Participation } \\
\text { Index }\end{array}$ \\
\hline EarlyImpact $(\mathrm{n}=245)$ & 21.1 & 15.25 & 19.48 \\
Effect size & $(1.14)$ & $(1.43)$ & $(1.25)$ \\
Mid Impact $(\mathrm{n}=129)$ & 11.30 & 8.32 & 10.51 \\
Effect size & $(1.68)$ & $(2.02)$ & $(1.46)$ \\
Late Impact $(\mathrm{n}=106)$ & & 7.47 & 9.1 \\
Effect size & 8.54 & $(1.99)$ & $(1.57)$ \\
\hline
\end{tabular}

${ }^{*} \mathrm{p}<0.001$ for each comparison. 
chronicity groups showed reduction in disability from admission to discharge. The greatest gains in functional independence were obtained by the EI group followed by the MI and LI groups respectively. At the completion of rehabilitation, functioning (as measured by the three MPAI-4 indices) was equivalent across chronicity groups. This finding is illustrated in Table 4, which shows mean T-scores and standard deviations for admission and discharge MPAI-4 indices across chronicity groups.

\section{Discussion}

Meaningful evaluation of TBI outcomes requires psychometrically sound metrics of performance. Without such measures, inferences concerning factors affecting the course of recovery following TBI are suspect. Consistent with prior research [9] [10], Rasch analyses performed at admission and discharge found the MPAI-4 to be a psychometrically acceptable measure of disability following TBI and a reasonable measure to evaluate effectiveness of post-hospital brain injury rehabilitation. Conversion of the Abilities, Adjustment, and Participation item scores to T-scores created interval scale metrics appropriate for use with powerful multivariate statistical models, lending greater confidence to the key findings.

The first of these findings was that the LI group on average was less severely impaired on the Abilities (physical and cognitive skills) and Participation (societal participation) indices than the EI and MI groups. The average chronicity of participants in the LI group was 9.9 years. Their lower admission T-scores (less disability) likely reflects the ability to adapt to environmental demands and compensate for impairments. Nonetheless, their admission scores were indicative of moderate-severe disability which precluded them from living independently.

Of considerable importance, the RM MANCOVA and follow-up comparisons revealed that each of the three chronicity groups made significant improvement on the MPAI-4 indices from admission to discharge. Despite large differences in chronicity, scores among the three groups on the dependent variables were not

Table 4. Means (Standard Deviations) for MPAI-4 indices at admission (ADM) and discharge (DC) by chronicity groups.

\begin{tabular}{ccccccc}
\hline \multicolumn{1}{c}{\begin{tabular}{c}
\multicolumn{7}{c}{ Chronicity Group } \\
Index
\end{tabular}} & \multicolumn{2}{c}{ Early Interval } & \multicolumn{2}{c}{ Mid Interval } & \multicolumn{2}{c}{ Late Interval } \\
\hline & ADM & DC & ADM & DC & ADM & DC \\
\hline \multirow{2}{*}{ Abilities } & 59.47 & 50.90 & 59.12 & 52.39 & 54.62 & 49.96 \\
& $(10.10)$ & $(10.20)$ & $(12.14)$ & $(13.68)$ & $(9.41)$ & $(10.96)$ \\
& 60.22 & 52.42 & 58.42 & 52.44 & 58.39 & 53.12 \\
Adjustment & $(9.78)$ & $(9.37)$ & $(9.98)$ & $(10.67)$ & $(9.55)$ & $(10.23)$ \\
& 59.49 & 50.79 & 57.41 & 51.96 & 54.74 & 49.69 \\
Participation & $(9.62)$ & $(10.26)$ & $(11.14)$ & $(11.98)$ & $(9.75)$ & $(9.61)$ \\
\hline
\end{tabular}


statistically different at discharge. As would be expected, the EI group showed the greatest improvement. Their injuries were more acute and as such would have a greater impact on skill performance, but they also would realize the greatest benefit from natural healing. The combination of these factors and comprehensive rehabilitation was likely responsible for the magnitude of improvement observed in this group. The MI group showed the second greatest improvement. With an average chronicity of 13 months, improvement was driven less by natural healing and more by events associated with the rehabilitation program. These participants also had less time to habituate poor habits ("negative plasticity") than those in the LI group, therefore, presenting fewer barriers to recovery.

Perhaps the most notable finding was the statistically significant improvement realized by the LI group on each of the three dependent variables. Improvement occurring at this late stage post-injury is an encouraging result for TBI survivors who must live with chronic disability. This finding, consistent with prior research by Lewis and Horn [3] and Hayden et al. [4], suggests that along with emotional support participation in physically and cognitively challenging activities can not only maintain functioning but can also reduce disability. These findings offer empirical evidence for the value of post-hospital rehabilitation for TBI survivors late in recovery. Moreover, results are consistent with brain plasticity research demonstrating that cortical reorganization and resulting functional change can occur years after injury [11].

\section{Conclusion}

Application of multivariate statistics to the study of functional outcomes following TBI was presented as an effective and efficient approach to gain a more precise and scientifically sound understanding of multivariate influences on performance. Multivariate analysis can only be appropriately used with intervalscale ("parametric equivalent") data. The Rasch model illustrated in this study supports the development of precise measurement with interval level metric qualities. Such measures enable the clear differentiation among persons measured along the disability continuum and are necessary to modify rehabilitative treatments for better functional outcomes.

\section{Limitations of the Study}

This study concluded at the time of program discharges from the various facilities. Long-term follow-up is needed to evaluate the durability of the changes achieved in program, and specifically determine whether maintenance of skills is equivalent across the chronicity groups. Also, the study design did not permit the identification of specific interventions responsible for the observed changes.

\section{References}

[1] Bond, M. (1983) Standard Methods of Assessing and Predicting Outcome. In: Rosenthal, M., Griffith, E.R., Bond, M. and Miller, J., Eds., Rehabilitation of the Head Injured Adult, F.A. Davis Co., Philadelphia, 97-113. 
[2] Nudo, R.J. and Dancause, N. (2007) Neuroscientific Basis for Occupational and Physical Therapy Interventions. In: Zasler, N.D., Katz, D.I. and Zafonte, R.D., Eds., Brain Injury Medicine: Principles and Practice, Demos Medical Publishing, New York, 913-928.

[3] Lewis, F. and Horn, G. (2013) Traumatic Brain Injury: Analysis of Functional Deficits and Post Hospital Rehabilitation Outcomes. Journal of Special Operations Medicine, 13, 56-61.

[4] Hayden, M.E., Plenger, P. and Bison, K. (2013) Treatment Effect versus Pretreatment Recovery in Persons with Acquired Brain Injury: A Study Regarding the Effectiveness of Post-Acuterehabilitation. American Academy of Physical Medicine and Rehabilitation, 4, 319-327.

[5] Merbitz, C., Morris, J. and Grip, J.C. (1989) Ordinal Scales and Foundations of Misinference. Archives of Physical Medicine and Rehabilitation, 70, 308-312.

[6] Grimby, G., Tennant, A. and Tesio, L. (2012) The Use of Raw Scores from Ordinal Scales: Time to End Malpractice? Journal of Rehabilitation Medicine, 44, 97. https://doi.org/10.2340/16501977-0938

[7] Kucukdeveci, A., Tennant, A., Grimby, G. and Franchignoni, F. (2011) Strategies for Assessment and Outcome Measurement in Physical and Rehabilitation Medicine: An Educational Review. Journal of Rehabilitation Medicine, 43, 661-672. https://doi.org/10.2340/16501977-0844

[8] Bond, T. and Fox, C. (2007) Applying the Rasch Model: Fundamental Measurement in the Human Sciences. $2^{\text {nd }}$ Edition, LEA, Mahwah, NJ.

[9] Malec, J. and Lezak, M. (2008) Manual for the Mayo-Portland Adaptability Inventory (MPAI-4) for Adults, Children and Adolescents. The Center for Outcome Measurement in Brain Injury. http://www.tbims.org/combi/mpai

[10] Malec, J., Kragness, M., Evans, R., Finlay, K., Kent, A. and Lezak, M. (2003) Further Psychometric Evaluation and Revision of the Mayo-Portland Adaptability Inventory in a National Sample. Journal of Head Trauma Rehabilitation, 18, 479-492. https://doi.org/10.1097/00001199-200311000-00002

[11] YouRong, S., Veeravagu, A. and Grant, G. (2016) Neuroplasticity after Brain Injury. In: Laskowitz, D. and Grant, G., Eds., Translational Research in Traumatic Brain Injury, CRC Press/Taylor and Francis Group, Boca Raton, FL.

Submit or recommend next manuscript to SCIRP and we will provide best service for you:

Accepting pre-submission inquiries through Email, Facebook, LinkedIn, Twitter, etc. A wide selection of journals (inclusive of 9 subjects, more than 200 journals)

Providing 24-hour high-quality service

User-friendly online submission system

Fair and swift peer-review system

Efficient typesetting and proofreading procedure

Display of the result of downloads and visits, as well as the number of cited articles

Maximum dissemination of your research work

Submit your manuscript at: http://papersubmission.scirp.org/

Or contact ojs@scirp.org 